\title{
Omega: An Architecture for AI Unification
}

\author{
Eray Özkural \\ Celestial Intellect Cybernetics \\ celestialintellect.com
}

\begin{abstract}
We introduce the open-ended, modular, selfimproving Omega AI unification architecture which is a refinement of Solomonoff's Alpha architecture, as considered from first principles. The architecture embodies several crucial principles of general intelligence including diversity of representations, diversity of data types, integrated memory, modularity, and higher-order cognition. We retain the basic design of a fundamental algorithmic substrate called an "AI kernel" for problem solving and basic cognitive functions like memory, and a larger, modular architecture that re-uses the kernel in many ways. Omega includes eight representation languages and six classes of neural networks, which are briefly introduced. The architecture is intended to initially address data science automation, hence it includes many problem solving methods for statistical tasks. We review the broad software architecture, higher-order cognition, self-improvement, modular neural architectures, intelligent agents, the process and memory hierarchy, hardware abstraction, peerto-peer computing, and data abstraction facility.
\end{abstract}

\section{Introduction and Motivation}

In today's AI research, most researchers focus on specific application problems and they develop the capabilities of their AI solutions only to the extent that these specific applications require them. While challenging AI problems such as natural language understanding require a broader view, most researchers do not begin with an all-encompassing architecture and then adapt to a specific application. It is usually more efficient to pursue a bottom-up development methodology for the experimental results, and as a result, progress in ambitious architectures for generality may have stalled.

To achieve generality, a rigorous architectural approach has several benefits such as easing development, allowing future extensions while remaining backwards compatible, and exposing problems before they happen since we can conceptualize complex use-cases. In other words, it is at least better software engineering, however, there are also scientific benefits such as understanding the functions and capabilities required by a general-purpose AI system much better, and address these problems fully. Since the most general problem is attacked, the architecture can follow a rigorous design process which will eliminate redundancies, leading us to a more mathematically elegant design. And finally, since use-cases will lead the design, the result will be empirically firmer than a special-purpose application.

A design from first principles is rarely undertaken, and it is arduous, but it can produce highly effective systems. We build upon the most powerful architectures for general AI, and then identify the requirements, from which we introduce refinements to the existing architectures, introducing new architectural ideas and incorporating new AI technologies in the process. The resulting deep technological integration architecture is a compact, scalable, portable, AI platform for general-purpose AI with many possible applications in wide domains.

\section{Design Principles for Generality}

In this section, we review the requirements of a general AI system, and from this vantage point we formulate design principles for constructing a general system.

A general AI system cannot contain any and all specific solutions in its memory, therefore it must equal the computer scientist in terms of its productive capacity of solutions. The requirement of a universal problem solver therefore is fundamental to any such design. Naturally, this implies the existence of Turing-complete programming languages, and a universal method to generalize - which implies a universal principle of induction such as Solomonoff induction. A suitably general probabilistic inference method such as Bayesian inference is implied since most AI problems are probabilistic in nature. It must have practically effective training methods for learning tasks, such as the GPU accelerated training methods used in deep learning. The system must have an integrated memory for cumulative learning. The architecture must be modular for better scalability and extensibility; human brain is a little like that as the neo- 
cortex has a grid of cortical columns, which are apparently functionally equivalent structures.

A general AI system must be able to support robotics, however, it should not be limited to agent architectures; it must also support traditional applications like databases, web search, and mobile computing. To accommodate for such a wide variety of functions, the architecture must expose a Swiss army knife like AI toolkit, to provide a unified AI API to developers. Such an API can then be served over the cloud, or via fog computing. Machine learning applications generally require hardware with high performance computing support. Therefore, the architecture should be compatible with high performance computing hardware such as GPUs, and FPGAs to be able to scale to many clients.

The general AI system must also address all the hard challenges of a natural environment as formulated by Russell and Norvig, 2016, Chapter 2]: the system must cope with the partially observable environments, multi-agent environments, competition and cooperation, stochastic environments, uncertainty, nondeterminism, sequential environments, dynamic environments, continuous environments, and unknown environments. A tall order, if there were ever one. Therefore, the system must be designed with these features of the environment in mind, for accommodating their needs.

AIXI Hutter, 2007 addresses partially observable environments, however, the rest of the features require architectural support in most cases, such as the necessity of providing a theory-theory module (a cognitive module that has a theory of other minds), or showing that the system will discover and adapt to other minds. To provide for multi-agent environments, the system can offer a self-simulation virtualization layer so that the agent can conceive of situations involving entities like itself. To support proper modeling of environments like with stochastic and uncertainty, we need an extensive probabilistic representation language to deal with non-trivial probabilistic problems; the language must cover common models such as hiearchical hidden markov layer models; it should offer a wide range of primitives to choose from, which must be supplied by the architecture. The representation language must also provide the means to combine primitives meaningfully, and obtain short programs for common patterns. The mystique art of designing compact representation languages therefore remains a vital part of AI research. To provide for effective representation of things like sequential, dynamic, continuous environments, the architecture can provide effective representation primitives and schemas. For dealing with unknown environments, the architecture can provide an agent architecture that can engage in the exploration of the unknown, much as an animal does.

Without doubt, the system must also accommodate common data types, and common tasks such as speech recognition, and the examples for more specific operations should be provided. It is important that the system allows one to implement a wide family of AI tasks for the system to be considered sufficiently general. If,

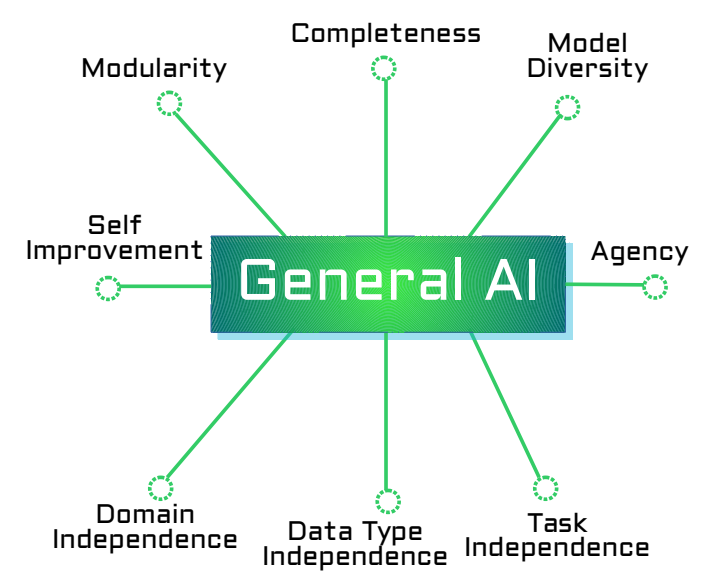

Figure 1: Some principles of general intelligence.

for instance, the user cannot feasibly implement something like style transfer, that is popular in deep learning research, with the architecture, it should rather not be termed general. The system should support a wide range of structured, and unstructured data, including popular data types like image, audio, video, speech, and text, and have sufficiently rich models to represent these challenging kinds of data. These more human data types constitute the primary means by which humans can communicate with AI's directly. However, structured, regular and irregular data types also must be supported, since these originate from a variety of sources that can be consumed by the AI system.

The system must also therefore provide an adequate perception architecture by which such a system can learn a world-representation from its sensorium which includes many senses. These processes should be sufficiently general that they can be adapted to any sort of sensorium that will work under known laws of physics. The system should also support an adequate intelligent agent architecture that supports typical goal following, or utility maximization architectures.

Therefore, it also is a challenge to test system generality. Typically, a benchmark that consists of a large number of diverse AI tasks and datasets must be provided for the system to demonstrate generality. The benchmark should be diverse enough to include the whole gamut of AI problems such as typical pattern recognition problems of image recognition, speech recognition, but also natural language understanding, machine learning tasks like anomaly detection (over real-world datasets such as an industrial dataset), time-series prediction (commonly used for stock market analysis), robotics problems, game playing problems, and so forth with randomly varied parameters.

We therefore arrive at an understanding of generalpurpose AI design that tries to maximize generality for every distinct aspect of a problem. The solution space must be wide enough to cover every problem domain. 
The methods must be independent from the data type. The tasks that can be performed should not be fixed. The system should be independent from the task to be solved; any task should be specifiable. The architecture must not depend either on a particular representation, it should cover a very wide range of representations to be able to deal with different kinds of environments. The intelligent agent code should not be environment specific, it must be adaptable to any environment and agent architecture; in other words, the system must be independent of the environment. Some principles of general intelligence are depicted in Figure 1 on page 2.

\section{Architecture Overview}

Many of the aforementioned problems have been addressed by existing AI architectures. We therefore take a well-understood general AI architecture called the Alpha architecture of Solomonoff Solomonoff, 2002, and define some basic capabilities better, while incorporating newer models and methods from recent research.

For the purposes of general-purpose AI, two most significant events have occured since Alpha was designed in 2002. First, the Gödel Machine architecture Schmidhuber, 2006 which also provides a level of self-reflective thinking, and presents an agent model around it. The other notable development is the immense success of deep learning methods, which now enable machines to achieve pattern recognition at human-level or better for many basic tasks. The present design therefore merges these two threads of developments into the Alpha framework. The architecture also provides for basic universal intelligent agents, and self-reflection like Gödel Machine does. Unlike Gödel Machine, we do not assume that the environment is known to a substantial degree, such things are assumed to be learnt.

Like the Alpha architecture, we assume a basic problem solver that is smart enough to bootstrap the rest of the system. This component is called the AI Kernel.

The system is thought to be parameter free, dependent only on the data, and the commands given. The system's interface is a graphical web-based application that allows the user to upload datasets and then apply AI tasks from the library. The system also provides an API for programming novel tasks. A basic graphical programming environment is considered for later releases since the system aims to be usable by non-programmers.

\subsection{Components}

We review the major components of the system architecture, and explain their functions.

\section{AI Kernel}

The AI kernel is an inductive programming system that should use a universal reference machine such as LISP. We have proposed using Church as the reference machine of such a system. However, what matters is that the AI kernel must be able to deal with all types of data, and tasks. We assume that the reference machine is variable in the right AI kernel. The kernel must be a com- pact code base that can run on a variety of hardware architectures to ensure portability, and the parallelization must support heterogeneous supercomputing platforms for high energy efficiency and scalability.

The AI kernel supports sophisticated programmability, allowing the user to specify most machine learning tasks with a very short API. We employ OCaml generic programming to characterize the kernel's internal components, model discovery, and transfer learning algorithms.

The AI kernel supports real-time operation, and can be configured to continuosly update long-term memory splitting running-time between currently running task and meta-learning.

\section{Bio-mimetic Search}

State-of-the-art bio-mimetic machine learning algorithms based on such methods as stochastic gradient descent, and evolutionary computation are available in the AI kernel, and thus chosen and used automatically.

\section{Heuristic Algorithmic Memory 2.0}

The AI kernel has integrated multi-term memory, meaning that it solves transfer learning problems automatically, and can remember solutions and representational states at multiple time scales. Heuristic Algorithmic Memory 2.0 extends Heuristic Algorithmic Memory Özkural, 2011 to support multiple reference machines.

\section{Problem Solvers}

Problem Solution Methods (PSMs) are methods that solve a given problem. These could be algorithmic solutions like sorting a list of numbers, or statistical methods like predicting a variable. The Alpha architecture basically tries a number of PSMs on a problem until it yields. However, in Omega, it is much better specified which PSMs the system should start with. Since the system is supposed to deal with unknown environments, we give priority to machine learning and statistical methods, as well model classes that directly address some challenging properties of the environment, and support hard applications like robotics. The diversity of the model classes and methods supported expand the range of Omega applications. The Alpha architecture can invent and retain new PSMs, that is why it should be considered an openended architecture; so is Omega.

The architecture is taught how to use a problem solver via unstructured natural language examples, like the intent detection task in natural language processing.

Both narrowly specialized and general-purpose methods are included in the initial library of problem solvers for initially high machine-learning capability.

For approximating functions, there are model-based learning algorithms like a generic implementation of stochastic gradient for an arbitrary reference machine. For model discovery, model-free learning algorithms like genetic programming are provided. Function approximation facilities can be invoked by the ensemble machine to solve machine learning problems. Therefore, a degree 
of method independence is provided by allowing multistrategy solvers.

A basic set of methods for solving scientific and engineering problems is provided. For computer science, the solutions of basic algorithmic problems including full software development libraries for writing basic computer programs for each reference machine (standard library). For engineering, basic optimization methods and symbolic algebra. In the ultimate form of the architecture, we should have methods for computational sciences, physical, and life sciences.

A full range of basic data science / machine learning methods are provided including:

Clustering Clustering is generalized to yield automated statistical modeling. Universal induction can be used to infer a PDF minimizing expected divergence (AI kernel function). Both general-purpose and classical clustering algorithms are provided, in recognition that for a specific class of problems a specialized method can be faster, if not necessarily more accurate. The classical algorithms of k-means Hartigan and Wong, 1979, hierarchical agglomerative clustering [Murtagh and Legendre, 2014], and Expectation Maximization (EM) for Gaussian Mixture Models $\mathrm{Xu}$ and Jordan, 1996 are provided. General-purpose algorithms based on NID |Vitányi et al., 2009], and universal induction enable working with arbitrary domains.

Classification Again both classical and generalpurpose algorithms are supported. Classical algorithms of decision-tree classifier Quinlan, 2014, random forest Liaw et al., 2002, knn Altman, 1992, logistic regression Cox, 1958, and SVM Vapnik et al., 1995 are supported. Generalpurpose algorithm invokes AI kernel universal induction routines to learn a mapping from the input to a finite set. NID based classifier works with arbitrary bitstrings.

Regression General-purpose algorithm invokes universal induction routines in the AI kernel to learn a stochastic operator mapping from the data domain to a real number. Classical algorithms of linear regression, logistic regression, and SVM are supported.

Outlier detection The generalized outlier detection finds the points least probable given the rest of the dataset using a generalization of z-score; to first model the data again a universal set induction invocation characterizes the data.

Time-series Forecasting Time series prediction is generalized with a universal induction approach modeling the stochastic dynamics, then the most probable model is inferred. Classical time-series prediction algorithms of ARIMA, Hidden Markov Model (HMM), and Hiearchical Hidden Markov Model (HHMM) Fine et al., 1998 are provided. A deep LSTM based forecast method is also provided
Hochreiter and Schmidhuber, 1997.

Deep Learning A complete range of DNN architectures for various data types such as image, audio, video and text are provided. Standard algorithms of backpropagation, stochastic gradient and variational inference are supported. The state-ofthe-art fully automated machine learning algorithm of Fourier Network Search (FNS) Koutník et al., 2014 is included. We also invoke universal induction routines to automate neural model discovery. The deep learning implementations are parallelized for multi GPU clusters. For this purpose, an existing deep learning framework such as TensorFlow may be used. The deep learning framework we use is a different, proprietary approach that predates TensorFlow and is composed of a neural programming language called MetaNet and a heterogeneous supercomputing middleware called Stardust.

Each algorithm mentioned is exposed as a PSM in the system.

\section{Ensemble Machine}

An ensemble machine is introduced to the system which runs PSMs in parallel with time allocated in accordance with their expected probability of success. The associations between tasks and their success are remembered as a stochastic mapping problem solved with the universal induction routines of AI Kernel, guiding future decisions. The ensemble machine itself is exposed as a PSM.

\subsection{Representation Languages}

We define eight reference machines to widen the range of solutions obtainable, and types of environments/applications addressable.

MetaNet MetaNet is a new General Neural Networks (GNN) representation language that encompasses common neuron types and architectures used in neural network research. It is a graphical metalanguage that can be used to define a large number of network architectures. Formally, it uses a multipartite labeled directed graph with typed vertices, as a generic representation to represent neural circuits, and the richer sort of representation allows us to extend the model to more biologically plausible, or with neuroscience-inspired models. The system uses this representation to facilitate automated model discovery of the right neural network for the given task when evaluating the MetaNet representation language.

Church We use the Church language Goodman et al., 2012 to represent probability distributions and solve basic algorithmic problems like adding a list of numbers, and the Towers of Hanoi problem. Components expose their interfaces in Church machine, expanding self-reflection capability.

Probabilistic Logic We define a probabilistic logic programming language to deal with uncertainty 
and stochasticity, and the ability to solve reasoning problems.

Bayesian Networks We define a general class of bayesian networks that can be used to deal with uncertainty.

Analog Computer We use an analog computing model to represent dynamical, continuous and stochastic systems better.

Picture We use the Picture Kulkarni et al., 2015a language to deal with images.

Matrix Computer We use an LAPACK based matrix algebra computing package such as GNU Octave to represent mathematical solutions.

Asynchronous Computer We define an asynchronous model of computation for conception of fine-grain concurrent models.

\subsection{Neural Representation Classes}

There are a number of ready neural representations that the system can quickly invoke.

Fourier Neural Network Fourier Neural Networks use a Fourier series representation to represent neural networks compactly, and may be considered a general-purpose learning model class Koutník et al., 2010b, Koutník et al., 2010a.

Convolutional Neural Networks CNNs are particularly effective for pattern recognition problems. A variety of basic CNNs [LeCun et al., 1995] suitable for processing different kinds of data are provided, including specialized networks such as multi-column DNNs for image classification Ciresan et al., 2012, for video Karpathy et al., 2014, text Zhang et al., 2015., and speech Abdel-Hamid et al., 2014.

Deep Belief Networks These networks are a stack of Restricted Boltzmann Machines Hinton et al., 2006. that can perform unsupervised learning.

Deep Autoencoders Deep autoencoders Hinton and Salakhutdinov, 2006. use several hidden unit layers, two deep belief networks, that learn to compress and then reproduce the data. We provide specific applications like variational autoencoders for image captions $\mathrm{Pu}$ et al., 2016, inverse graphics Kulkarni et al., 2015b, multimodal learning Ngiam et al., 2011.

LSTM/GRU networks We provide a variety of RNN models using LSTM (Long-Short Term Memory) and GRU (Gated Recurrent Unit) stacks to model sequential data. Variants for different data types such as speech Graves and Jaitly, 2014, video Zhang et al., 2016, image Donahue et al., 2015 are included.

Recursive Deep Networks Especially useful for language processing, these networks can recognize hierarchical structures easily Socher et al., 2013].
The networks are specified as generic network architectures that can scale to required input/output size. Any hyper-parameters are designated as variables to be learned to the AI kernel so that the hyper parameters can adapt to the problem. These networks are considered to be sufficient as providing enough library primitives. The generators for neural networks are specified such that the program generator can indeed generate all of the library networks; however, re-inventing the wheel is not a feasible idea, therefore we aim to include a complete inventory of deep learning models.

\subsection{Software Architecture}

\section{Functional Decomposition}

A high-level component architecture without the many inter-component interactions is depicted in Figure 2 on page 6 .

The system's process flow is straightforward. The user presents the system with a number of datasets, and the user selects a task to be applied to the data. The system automatically recognizes different data types, however, it also allows data to be specified in detail by a description language. The system will also accept tasks to be defined via a conversational engine, and a programming interface (API). The conversational engine can learn to recognize a task via given examples, mapping text to a task specification language and backwards. The programming interface accumulates the interfaces of all the components, unified under a single facade of a generic problem solver, which is formulated as a general optimizer Alpcan et al., 2014. As in Alpha, the most general interface the system provides is that of time-limited optimization, however, the system allows to solve any well-defined problems allowing the user to define any success criterion. The problem solver then predicts the probability that a PSM will succeed in solving the sospecified problem, and then translates the input data and the task to a format that the particular PSM will understand, and also translate any results back. After a task is solved, the system automatically updates its long-term memory and writes a snapshot to the disk. It then executes higher-order cognition routines to improve its PSMs, and awaits for the next task.

\section{Execution}

The execution of PSMs is parallelized as much as possible, as many PSMs may be run in parallel, but also some methods will allow data to be sharded, and will also parallelize well themselves. A main operational goal of the system is the ability to keep track of these parallelizations well enough to present an OS like stability to the user with a simple interface. The system also allows modules to be invoked concurrently and in a distributed manner to facilitate the design of distributed and decentralized applications using the API.

The PSMs are executed with a hardware abstraction layer called Stardust that provides heterogeneous peer-to-peer computing capability to the architecture. MetaNet acts as a common neural network representa- 


\section{Q. Architecture Eomponents}

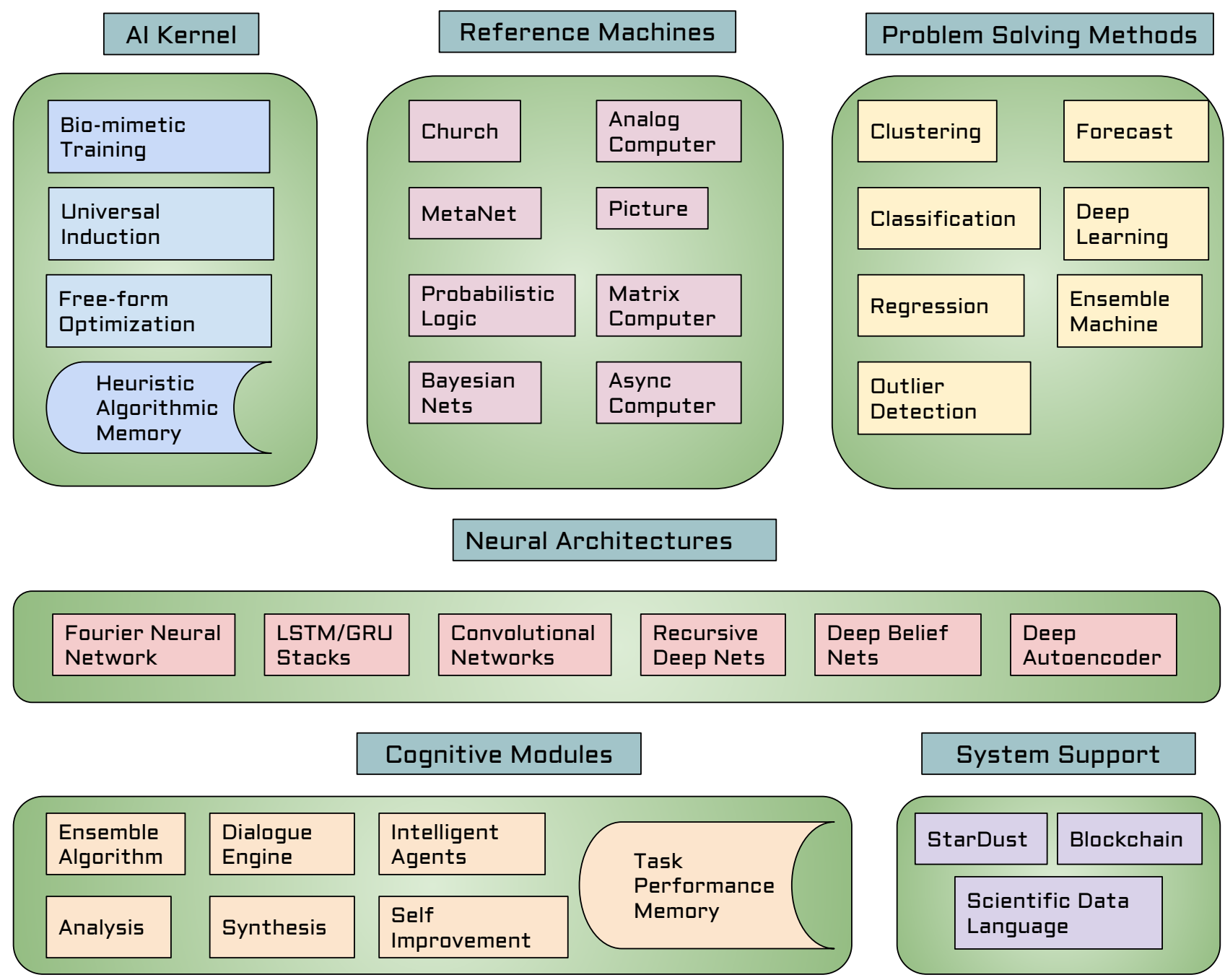

Figure 2: $\Omega$ Architecture Component Diagram 
tion language. Scientific Data Language is a data specification language that allows us to describe the type, format and semantic labels of the data.

\subsection{Higher-Order Cognition}

Two fundamental higher-order cognitive functions are defined as analysis and synthesis. Analysis decomposes a problem into components and then tries to solve the problem by first solving sub-problems and then merging their results into a solution. Synthesis generates new PSMs by combining known PSMs. These operations give the ability to observe the code of its modules, and expand the system's repertoire of PSMs continuously. Analysis is self-reflective in that sense, and synthesis is self-reification.

These functions correspond to a second kind of modularity where the tasks themselves can be decomposed, and entirely new PSMs may be invented and added as new modules to the system.

The system continually self-reflects through updating its algorithmic memory for accelerating future solutions. It also keeps a record of task performance for trying to retroactively optimize past solutions. The components expose themselves via a high-level reference machine (Church) which acts as the system "glue code" to compose and decompose system functions. Since Church is quite expressive, it can also act as the system's task description code, and be used to recognize, decompose, and compose tasks and solutions. The synthesis and analysis modules operate over the system's modular cognition itself, helping with synthesis of new solution methods and analysis of problems. The system uses self-models to guide its self-improvement, for instance, by trying to optimize its performance.

\subsection{Self-Improvement}

Analysis and synthesis can learn how to accomplish this as they can use the execution history to improve the results retrospectively. After a new problem is solved, therefore, the system can continuously try to improve its consolidated memory of PSMs by trying to generate new PSMs that will improve performance over history, or by decomposing problems to accelerate their execution. A general objective such as maximizing energy efficiency of solutions can be sought for self-improvement.

\subsection{Modular Neural Architectures}

PSMs embody a basic kind of modularity in the system which are extended with modular neural architectures. These architectural schemas are a cortical organization that decomposes the networks into many cortical columns, which are henceforth again decomposed into micro-columns, with variant geometries. This organization schema is called MetaCortex, and it is a way to describe larger networks that can digest a variety of data sources, and construct larger neural models with better modularity, that is better data/model encapsulation based on affinity. There are architectures such as multi-column committee networks that already implement these architectures, however, we would expand this to the entire library of networks described.

\subsection{Intelligent Agents}

Basic goal-following and utility-maximization agents can be realized similarly to time-series prediction. A typical two part model of learning representations (world model), and planning will be provided. A basic neural template will provide for multi-modal perception, multi-tasking, task decomposition and imitation learning. Neural templates corresponding to different kinds of agents such as Deep Mind's I2A model Weber et al., 2017. will be provided.

The intelligent agents have a real-time architecture, they run at a fixed number of iterations every second. At this shortest period of synchronization, mostly backpropagation like learning algorithms, and simulation are allowed to complete. Everything else is run in the background for longer time-scales.

\subsection{Process and Memory Hierarchy}

The processes and memory are organized hierarchically from long-term, heavy tasks to short-term, lightweight tasks. At the shortest scale, the system has neural memory units like LSTM, that last at the scale of one task, and model-based local training/inference algorithms like backpropagation algorithms. At a longer scale which corresponds to one iteration of problem solution procedure, the system remembers the best solutions so far, and it updates its mid-term memory with them to improve the solution performance in the next iteration. At this scale, the system will also engage in more processes such as the just mentioned memory update operation, and more expensive training algorithms such as genetic algorithms. At the highest scale, the system runs the most expensive model-free learning algorithms that can search over architectures, models, and components, and updates its persistent, long term memory based on the statistics about solutions of the new problem after solving it to guide the solution of new problems. The system also updates its PSMs by executing its higher-order cognitive functions at this scale.

\subsection{Hardware Abstraction Layer}

The architecture depends on a Hardware Abstraction Layer (HAL) in the form of Stardust peer-to-peer computing substrate. Stardust provides a bytecode representation that can be run on both multi-core cluster, GPU clusters, and FPGA clusters in the future. Stardust uses virtualization technology for compartmentalization and basic security. It uses a lightweight kernel, and provides parallel and distributed computing primitives.

\subsection{Peer-to-Peer Computing}

Peer-to-peer computing is facilitated by a node software that users download and operate to earn fees from the network with a cryptographic utility token. Since approaching human-level will typically require several 
petaflops/sec of computing speed, scaling to a significant number of global users requires peer-to-peer computing. If a proportion of profit is paid to the users, this can incentivize their contribution, providing a cost-effective computing platform for the architecture.

\subsection{Scientific Data Language}

A canonical data representation seems essential for Alpha family of architectures, because the PSMs can vary wildly in their assumptions. That is why, a common data format is required. The format we propose has standard representations for both structured (tabular, tree, network, etc.), unstructured (like text, audio, image, video) and complex data types. It supports web, cloud and fog computing data sources, thus also abstracting data ingestion. Each PSM handles the data differently, mapping to an internal representation if necessary. Therefore, every element of the data can be given a type, the format of the data may be specified (such as a 10x10 table of integers), and semantic labels may be ascribed to data elements. For instance, each dataset has a different domain, which may be designated with a domain path. Likewise, the physical units, or other semantic information may be annotated on a dataset with arbitrary attributes. The specification language must be also modular allowing to include other modules. The system automatically ingests known data formats, recognizes them, and converts into this common format which may henceforth be modified by the user. In the future, we are planning to design a data cleaning facility to improve the data at this stage.

\section{Discussion and Research Program}

We gave the overview of an ambitious architecture based on Solomonoff's Alpha Architecture, and Schmidhuber's Gödel Machine architecture. The system is like Alpha, because it re-uses the basic design of PSMs. It is also similar to Gödel Machine architecture, because it can deploy a kind of probabilistic logical inference for reasoning and it can also observe some of its internal states and improve itself. The system also has basic provisions for intelligent agents, but it is not limited to them. We saw that the first important issue with implementing Alpha was to decide a basic set of primitives that will grant it sufficient intelligence to deal with human-scale problems. It remains to be demonstrated empirically that is the case, however, two of the eight reference machines have been implemented and seen to operate effectively.

A criticism may be raised that we have not explained much about how the AI Kernel works. We only assume that it presents a generalized universal induction approximation that can optimize functions, rich enough to let us define basic machine learning tasks. It surely cannot be Levin search, but it could be any effective multi-strategy optimization method such as evolutionary architecture search Liang et al., 2018. We are using an extension of the approach in Fourier Network Search Koutník et al., 2010a which is also likely general enough. The memory update is also not detailed but it is assumed that it is possible to extend an older memory design called heuristic algorithmic memory so that it works for any reference machine. We also did not explain in detail how many components work due to lack of space, which is an issue to be tackled in a longer future version of the present paper.

In the future, we would like to support the architectural design with experiments, showing if the system is imaginative enough to come up with neural architectures or hybrid solutions that did not appear to humans. The algorithms used are expensive, therefore they might not work very well with the extremely large models required by the best vision processing systems; but to accommodate such models, it might be required that the system evolves only parts of the system and not the entire architecture. The system is intended to be tested on basic psychometric tests first, and a variety of data science problems to see if we can match the competence of the solution a human data scientist would achieve.

\section{References}

[Abdel-Hamid et al., 2014] Ossama Abdel-Hamid, Abdel-rahman Mohamed, Hui Jiang, Li Deng, Gerald Penn, and Dong Yu. Convolutional neural networks for speech recognition. IEEE/ACM Transactions on audio, speech, and language processing, 22(10):1533-1545, 2014.

[Alpcan et al., 2014] Tansu Alpcan, Tom Everitt, and Marcus Hutter. Can we measure the difficulty of an optimization problem? In 2014 IEEE Information Theory Workshop, ITW 2014, Hobart, Tasmania, Australia, November 2-5, 2014, pages 356-360, 2014.

[Altman, 1992] Naomi S Altman. An introduction to kernel and nearest-neighbor nonparametric regression. The American Statistician, 46(3):175-185, 1992.

[Ciresan et al., 2012] D. C. Ciresan, U. Meier, J. Masci, and J. Schmidhuber. Multi-column deep neural network for traffic sign classification. Neural Networks, 32:333-338, 2012.

[Cox, 1958] David R Cox. The regression analysis of binary sequences. Journal of the Royal Statistical Society. Series B (Methodological), pages 215-242, 1958.

[Donahue et al., 2015] Jeff Donahue, Lisa Anne Hendricks, Sergio Guadarrama, Marcus Rohrbach, Subhashini Venugopalan, Kate Saenko, and Trevor Darrell. Long-term recurrent convolutional networks for visual recognition and description. In CVPR, 2015.

[Fine et al., 1998] Shai Fine, Yoram Singer, and Naftali Tishby. The hierarchical hidden markov model: Analysis and applications. Machine learning, 32(1):41-62, 1998.

[Goodman et al., 2012] Noah Goodman, Vikash Mansinghka, Daniel M Roy, Keith Bonawitz, and Joshua B Tenenbaum. Church: a language for generative models. arXiv preprint arXiv:1206.3255, 2012. 
[Graves and Jaitly, 2014] Alex Graves and Navdeep Jaitly. Towards end-to-end speech recognition with recurrent neural networks. In Proc. 31st International Conference on Machine Learning (ICML), pages 1764-1772, 2014.

[Hartigan and Wong, 1979] John A Hartigan and Manchek A Wong. Algorithm as 136: A k-means clustering algorithm. Journal of the Royal Statistical Society. Series C (Applied Statistics), 28(1):100-108, 1979.

[Hinton and Salakhutdinov, 2006] Geoffrey E Hinton and Ruslan R Salakhutdinov. Reducing the dimensionality of data with neural networks. science, 313(5786):504-507, 2006.

[Hinton et al., 2006] Geoffrey E. Hinton, Simon Osindero, and Yee-Whye Teh. A fast learning algorithm for deep belief nets. Neural Computation, 18(7):15271554, May 2006.

[Hochreiter and Schmidhuber, 1997] S. Hochreiter and J. Schmidhuber. LSTM can solve hard long time lag problems. In M. C. Mozer, M. I. Jordan, and T. Petsche, editors, Advances in Neural Information Processing Systems 9 (NIPS 9), pages 473-479. MIT Press, 1997.

[Hutter, 2007] Marcus Hutter. Universal algorithmic intelligence: A mathematical top $\rightarrow$ down approach. In B. Goertzel and C. Pennachin, editors, Artificial General Intelligence, Cognitive Technologies, pages 227290. Springer, Berlin, 2007.

[Karpathy et al., 2014] Andrej Karpathy, George Toderici, Sanketh Shetty, Thomas Leung, Rahul Sukthankar, and Li Fei-Fei. Large-scale video classification with convolutional neural networks. In IEEE Conference on Computer Vision and Pattern Recognition (CVPR), 2014.

[Koutník et al., 2010a] J. Koutník, F. Gomez, and J. Schmidhuber. Evolving neural networks in compressed weight space. In Proceedings of the 12th Annual Conference on Genetic and Evolutionary Computation, pages 619-626, 2010.

[Koutník et al., 2010b] J. Koutník, F. Gomez, and J. Schmidhuber. Searching for minimal neural networks in Fourier space. In Proceedings of the 4th Annual Conference on Artificial General Intelligence. Atlantis Press, 2010.

[Koutník et al., 2014] Jan Koutník, Faustino Gomez, and Jürgen Schmidhuber. Evolving deep/recurrent networks for reinforcement learning. In Proceedings of the 2014 Genetic and Evolutionary Computation Conference (GECCO-2014). ACM Press, 2014. To appear.

[Kulkarni et al., 2015a] Tejas D Kulkarni, Pushmeet Kohli, Joshua B Tenenbaum, and Vikash Mansinghka. Picture: A probabilistic programming language for scene perception. In Proceedings of the ieee conference on computer vision and pattern recognition, pages 4390-4399, 2015.
[Kulkarni et al., 2015b] Tejas D Kulkarni, William F. Whitney, Pushmeet Kohli, and Josh Tenenbaum. Deep convolutional inverse graphics network. In C. Cortes, N. D. Lawrence, D. D. Lee, M. Sugiyama, and R. Garnett, editors, Advances in Neural Information Processing Systems 28, pages 2539-2547. Curran Associates, Inc., 2015.

[LeCun et al., 1995] Yann LeCun, Yoshua Bengio, et al. Convolutional networks for images, speech, and time series. The handbook of brain theory and neural networks, 3361(10):1995, 1995.

[Liang et al., 2018] Jason Liang, Elliot Meyerson, and Risto Miikkulainen. Evolutionary architecture search for deep multitask networks. arXiv preprint arXiv:1803.03745, 2018.

[Liaw et al., 2002] Andy Liaw, Matthew Wiener, et al. Classification and regression by randomforest. $R$ news, 2(3):18-22, 2002.

[Murtagh and Legendre, 2014] Fionn Murtagh and Pierre Legendre. Ward's hierarchical agglomerative clustering method: which algorithms implement ward's criterion? Journal of classification, 31(3):274295, 2014.

[Ngiam et al., 2011] Jiquan Ngiam, Aditya Khosla, Mingyu Kim, Juhan Nam, Honglak Lee, and Andrew Y Ng. Multimodal deep learning. In Proceedings of the 28th international conference on machine learning (ICML-11), pages 689-696, 2011.

[Özkural, 2011] Eray Özkural. Towards heuristic algorithmic memory. In $A G I$, pages 382-387, 2011.

[Pu et al., 2016] Yunchen Pu, Zhe Gan, Ricardo Henao, Xin Yuan, Chunyuan Li, Andrew Stevens, and Lawrence Carin. Variational autoencoder for deep learning of images, labels and captions. In D. D. Lee, M. Sugiyama, U. V. Luxburg, I. Guyon, and R. Garnett, editors, Advances in Neural Information Processing Systems 29, pages 2352-2360. Curran Associates, Inc., 2016.

[Quinlan, 2014] J Ross Quinlan. C4. 5: programs for machine learning. Elsevier, 2014.

[Russell and Norvig, 2016] Stuart J Russell and Peter Norvig. Artificial intelligence: a modern approach. Malaysia; Pearson Education Limited,, 2016.

[Schmidhuber, 2006] J. Schmidhuber. Gödel machines: Fully self-referential optimal universal self-improvers. In B. Goertzel and C. Pennachin, editors, Artificial General Intelligence, pages 199-226. Springer Verlag, 2006. Variant available as arXiv:cs.LO/0309048.

[Socher et al., 2013] Richard Socher, Alex Perelygin, Jean $\mathrm{Wu}$, Jason Chuang, Christopher D Manning, Andrew Ng, and Christopher Potts. Recursive deep models for semantic compositionality over a sentiment treebank. In Proceedings of the 2013 conference on empirical methods in natural language processing, pages 1631-1642, 2013. 
[Solomonoff, 2002] Ray J. Solomonoff. Progress in incremental machine learning. In NIPS Workshop on Universal Learning Algorithms and Optimal Search, Whistler, B.C., Canada, December 2002.

[Vapnik et al., 1995] Vladimir Vapnik, Isabel Guyon, and Trevor Hastie. Support vector machines. Mach. Learn, 20(3):273-297, 1995.

[Vitányi et al., 2009] Paul MB Vitányi, Frank J Balbach, Rudi L Cilibrasi, and Ming Li. Normalized information distance. In Information theory and statistical learning, pages 45-82. Springer, 2009.

[Weber et al., 2017] Théophane Weber, Sébastien Racanière, David P Reichert, Lars Buesing, Arthur Guez, Danilo Jimenez Rezende, Adria Puigdomènech Badia, Oriol Vinyals, Nicolas Heess, Yujia Li, et al. Imagination-augmented agents for deep reinforcement learning. arXiv preprint arXiv:1707.06203, 2017.

[Xu and Jordan, 1996] Lei Xu and Michael I Jordan. On convergence properties of the em algorithm for gaussian mixtures. Neural computation, 8(1):129-151, 1996.

[Zhang et al., 2015] Xiang Zhang, Junbo Zhao, and Yann LeCun. Character-level convolutional networks for text classification. In C. Cortes, N. D. Lawrence, D. D. Lee, M. Sugiyama, and R. Garnett, editors, $A d-$ vances in Neural Information Processing Systems 28, pages 649-657. Curran Associates, Inc., 2015.

[Zhang et al., 2016] Ke Zhang, Wei-Lun Chao, Fei Sha, and Kristen Grauman. Video summarization with long short-term memory. In ECCV, 2016. 